\title{
Feasibility of an at home, online, yoga-based and standard exercise intervention for rotator cuff injuries
}

\begin{abstract}
This study investigates the feasibility of conducting a 6-week intervention program comparing online yoga-based and standard exercises among 10 injured pre-surgery rotator cuff patients. A feasibility study of 10 patients recruited from surgical wait lists was conducted. Patients were assessed for shoulder flexion, abduction and external rotation range of motion and strength at baseline and 6-weeks. The SPAD questionnaire was used to assess pain/function. 8/10 patients did not complete the full study protocol. The case report illustrates that the yoga group patient worsened, while the standard exercise group patient improved in strength. SPADI results were changed by less than $10 \%$ indicating no clinically important change. Qualitative feedback suggested that patients might be open to online exercises however further developmental work is needed to define a feasible protocol.
\end{abstract}

Volume 2 Issue I - 2017

\author{
Dolly Mehta' Joy MacDermid' Jackie Sadi' \\ Ken Faber ${ }^{2}$ George Athwal ${ }^{2}$ \\ 'Department of Health and Rehabilitation Sciences, University \\ of Western Ontario, Canada \\ ${ }^{2}$ Department of St. Joseph's Hospital, Canada
}

Correspondence: Dolly Mehta, Department of Health and Rehabilitation Sciences, The University of Western Ontario, Canada,Tel 416-319-1900,Email twinkle_5000@hotmail.com

Received: November II, 2016 | Published: March 03, 2017

Keywords: yoga, rotator cuff, online exercises, rehabilitation

Abbreviations: RC, rotator cuff; RCI, rotator cuff injury; Std. Ex, standard exercises

\section{Introduction}

Rotator cuff (RC) tears are a very common orthopedic condition Reports claim that $25 \%$ of people in their 60 s and $50 \%$ of people in their 80 s suffer a RC tear, making it the most common shoulder surgery performed. ${ }^{1}$ Also, it is reported that the annual cost of RC repairs is approximately $\$ 3$ billion in the United States alone. ${ }^{2}$

Exercise has been shown to benefit RC patients in terms of reducing pain, improving strength, ROM and function. ${ }^{3}$ However, clinical studies have primarily examined the role of traditional exercise for $\mathrm{RC}$ rehabilitation. The effects of yoga as exercise through an online home based setting have been scarcely investigated. This chapter will discuss the feasibility of a 6 -week long exercise intervention. The purposes of this feasibility study were to:

i. Determine the logistics of implementing a 6 week, online exercises program for patients with rotator cuff (RC) tears awaiting surgical repair.

ii. Identify potential positive and negative patient responses to the exercise programs (treatment effects and harms).

iii. Identify recruitment issues and retention of participants for a future clinical trial.

iv. Identify the feasibility of implementing outcome measures.

v. Identify potential improvements in the research protocol that might be needed to move forward with the future clinical trial.

\section{Participants}

\section{Sample recruitment}

Patients were recruited by telephone from the surgical wait lists of two orthopedic surgeons (fourth and fifth co-authors) at the Roth McFarlane Hand and Upper Limb Centre (HULC) located in St. Joseph's Hospital. Patients provided informed consent as approved by their signatures on consent forms. The initial intention was to recruit a sample of up to 30 participants, assuming a $50 \%$ response rate and a potential pool of 60 participants that would be contacted within a threemonth interval. Over the course of the $3^{\text {rd }}$ month recruitment period 51 patient files were obtained from two surgical wait lists. Each file was analyzed to see if patients were eligible if their surgery was scheduled within 6 weeks from the time the file was received that patient was excluded. A total of 49 calls were made during those 3 months. (Two files did not have patient contact information so calls could not be made). Of 49 calls seven were excluded because the patient informed the examiner he/she would either be having their surgery within 6 weeks or had already undergone their surgery. Thus 42 patients were eligible however 32 declined the invitation to participate and only 10 consented. Of these 10 patients three were randomized to the standard exercise group and seven to the yoga group via envelopes. By the end of the 6 weeks only two patients completed the entire intervention. (One patient discontinued with the exercises because of her upcoming RC surgery while another patient opted out due to vacation. Three patients elected out due to pain. Finally three patients only provided qualitative feedback in response to predetermined interview questions over the telephone to inform understanding on their perceptions and adherence to the assigned exercise program since they did not underdog pre/post). In the end, two patients completed the program and the full pre/post testing (Figure 1) 


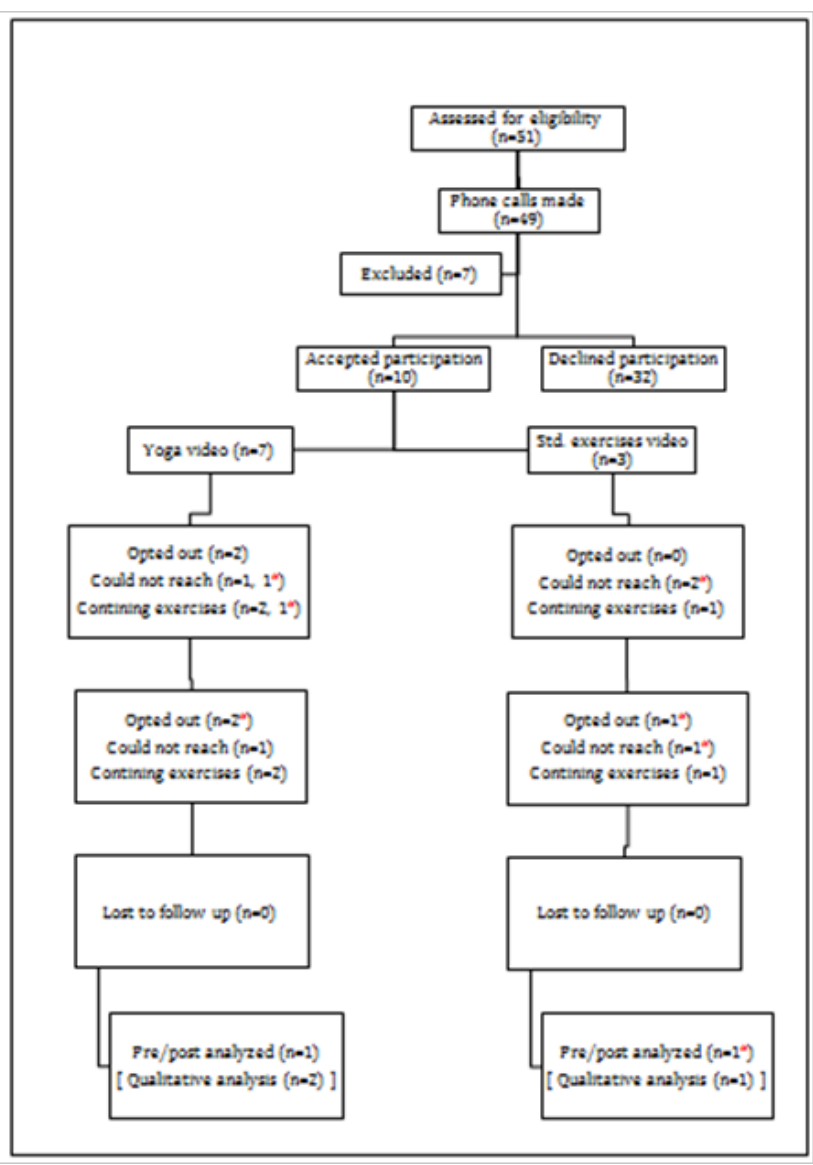

Figure I Flowchart demonstrating patient inclusion/exclusion.

\section{Methods}

\section{Inclusion/exclusion criteria}

To be included in the study patients had to be on a surgery wait list for their rotator cuff injury and be 18 years or older. Patients were excluded if their surgery was booked within 6 weeks or they had comorbidities that precluded participation in an exercise program.

\section{Ethics and consent}

A letter of information a patient information/consent form, a Diary form and a telephone script were created for this study. Ethical approval was obtained from Western University's Research Ethics Board.

\section{Development of interventions}

Standard exercise for rotator cuff is therapeutic exercise that recruits specific rotator cuff muscle groups. The comparison was a yoga-based exercise also intended to recruit rotator cuff muscles. It was decided to only focus on three exercises to optimize adherence and understanding given that this was an independent home program. Further, we purposively kept the number of exercises ${ }^{4}$ repetitions ( 3 sets, 10 repetitions) and length of instruction (under 5 minutes) the same in both exercise options. The second and third co-authors reviewed AAOS practice guidelines for home programs for patients with rotator cuff disorders integrated that with their own clinical expertise and identified three key therapeutic exercises standing row, internal rotation and external rotation. The physical therapist had experience with yoga and identified three yoga poses that might recruit rotator cuff muscles isometric prayer pose, half cobra pose and sphinx pose.

Once the exercises were selected a video script was created, reviewed and edited by all authors. During filming the third coauthor served as the instructor in the standard exercises video while a certified yoga teacher $(\mathrm{KN})$ served as the instructor for the yoga video. Both videos were shot using a Nikon point and shoot camera and edited on Movie by one co-author (DM) and reviewed by all coauthors. Revisions of the video were made based on co-author feedback. Both videos were kept under 5 minutes each so patient did not have to spend a long time following the video. Next videos were uploaded to YouTube as unlisted and patients were sent a link to their email addresses (which they provided during pre-assessments or through phone) for individual viewing. Only patients with the link could access the content. The unlisted option (compared to private) allowed participants to have access to the video without having a YouTube account and also remain closed so only the individuals who had the video link could access the content.

\section{Study measures}

The SPADI is a 13 item questionnaire that is used to measure pain and disability five questions evaluate pain and eight questions evaluate disability. ${ }^{4}$ SPADI is a reliable shoulder questionnaire for measuring $\mathrm{RC}$ disease ${ }^{5}$ acceptable for clinical practice and shows good construct validity. ${ }^{6}$ The minimally detectable change (MDC), which is the smallest real change outside of measurement error ${ }^{7}$ is 18 points $(95 \%)$ for patients assessed twice in SPADI. ${ }^{8}$ ROM was measured using a double-armed, clear plastic goniometer (Stryker Physiotherapy Associates) and strength using a HHD (JTECH Medical), a portable ${ }^{9}$ non-invasive, light-weight and efficient device that is capable of detecting minor increases or decreases in muscle strength. ${ }^{10}$ Compared to Manual Muscle Testing (MMT) the HHD is better because it provides a more objective evaluation in determining muscle strength. ${ }^{8}$

\section{Study procedures}

Participants were invited to HULC where ROM and strength testing were assessed. SPADI questionnaires were also completed during both pre and post. Consent forms were signed (one copy was given to the patient while another was kept in the clinic) and the study was explained in detail. Personal information including name, age, sex, rotator cuff history and location of injury (right or left shoulder) were noted by hand. Patients were then randomized via envelopes to receive either an online yoga or a standard exercises video. They were informed to which group they belonged immediately after randomization.

Clear instructions were given that patients had to do the movements to the best of their ability if it was too painful they could stop. Patients sat on a chair with their back against the frame. Strength levels were generally assessed first by the examiner using a HHD. The examiner demonstrated SF, SA and ER before having the patient perform the movements. ${ }^{6}$ The same testing protocol was used in both pre and post assessments. 


\section{Strength assessment}

Strength was measured using a HHD. All patients were assessed while seated. Calibration of the HHD and an explanation of the testing procedure were completed prior to commencing the test. For SF the patient had their elbow extended and at 90 degrees flexion. ${ }^{11}$ In our case, the patient had their elbow flexed at 0 degrees because it is likely that patients would have had difficulty starting at 90 degrees seeing as how their injury affected their ROM and strength. The HHD was placed above the patient's elbow ${ }^{12}$ or middle of the humerus. The examiner stood to the patient's testing side and held the HHD firmly in both hands. ${ }^{13}$

For SA the patient had their elbow fully extended and their arm at 90 degrees abduction..$^{13}$ Again, however, in our case the patient had their arm at 0 degrees for the mentioned reason. The HHD was placed in the same position as stated in SF. For ER the patient had their elbow flexed to 90 degrees arm at 0 degrees and forearm in neutral. Instructions to keep their elbow close to their body were given so arm abduction was avoided. The examiner placed the HHD on the "dorsal aspect of the distal forearm" and stood to the testing side clasping the device firmly in hand. ${ }^{13}$ Patients were instructed to exert maximum effort to move their arm in the required position while the examiner applied increasing resistance. ${ }^{14}$ They were also asked to sustain the effort for approximately 6 seconds until the examiner said to relax. ${ }^{15}$

Unfortunately, the isometric testing performed was compromised because patient's testing positions were not accurately controlled. They were asked to perform each movement to whichever degree they could before it began to hurt and they could not go any further. Each movement's range ended on a different arc. To illustrate SF, SA and ER all started at 0 degrees but there was no fixed point the patient was told to end their movement (i.e. stop at 90 degrees) which weakened the comparison of pre/post results. Unfortunately, with strength testing even minor changes in body positions significantly affected results ${ }^{16}$ so accuracy was compromised.

\section{ROM assessment}

All patients were tested while seated. (Both strength and ROM testing used the same chair). SF, SA and ER movements were measured using a goniometer. The advantages of goniometry allow for: effortlessness of use, direct measurement of shoulder joint angles cost, effective/economic benefit and portability. Unfortunately despite the advantages a major disadvantage is the manual inspection of goniometric reading. Results could only be visually estimated which made it difficult to assess ROM when measuring joints with thick layers of soft tissue. ${ }^{17}$ Typically, ROM testing occurred after strength testing however there was no fixed rule.

For SF and SA, the fulcrum or pin of the goniometer was closely aligned with the patient's glenohumeral joint axis. ${ }^{18}$ The center of the goniometer was placed on the posterior glenohumeral joint ${ }^{19}$ with one arm perpendicular to the floor and the other aligned according to the angle of the proximal humerus. ${ }^{20}$ For ER, the patient had their elbow flexed at 90 degrees with their humerus on the side. ${ }^{20}$ The goniometer was positioned with the fulcrum at the olecranon (under the elbow) with one arm parallel to the patient's thigh and the other moving along according to the forearm. ${ }^{21}$ Goniometric readings of maximum ROM were recorded for the affected and unaffected arm. ROM measures were taken only once for a total of six scores.

A single examiner (DM) performed all testing. It is important to note that the examiner is not a physiotherapist however testing was performed in the presence of an experienced physiotherapist, who verified the accuracy of the readings and provided suggestions for improvement. Finally t-tests were not performed because this is a feasibility study and it did not have enough power. Descriptive analysis was performed from both ROM and strength assessment.

\section{Follow up calls}

Patients enrolled in the study were informed they would receive follow-up calls approximately every 2 weeks to determine how the study was progressing. The primary question asked was how are you finding the exercises? During the first follow-up call (2-week mark) four out of 10 patients $(\mathrm{RC} 1 / 3 / 4 / 6)$ stated that they never received the video three patients $(\mathrm{RC} 1 / 7 / 8)$ did not answer the call so a brief voicemail message was left. (One patient (RC2) out of these three soon after returned the message and stated everything was going well [yoga group]). One patient (RC5) opted out due to pain with the (yoga) exercises and the remaining two patients (RC9/10) stated the exercises were "good" and "pretty good".

It is unknown why some patients did not receive the video link while others did. Nevertheless, video links were re-sent using HULC's email address instead of the examiner's Western's email account which was used initially. The four patients who did not receive the video links initially were called to see how they were progressing 2 weeks after video links were resent. (One out of these four patients (RC3) had informed the examiner of her hesitation with performing the (yoga) exercises due to limited mobility/strength and opted out). One patient $(\mathrm{RC} 1)$ did not answer the call so a voicemail message was left, another (RC4) stated he probably would not be continuing with the (yoga) exercises for much longer due to pain but would continue for a bit and the last patient (RC6) was waiting for a resistive band in the mail (which was required for the std. ex. video) and therefore had not begun the exercises.

During the 4 week follow up call for the patients who received the video when it was first sent one patient's (RC2) family member answered the call and took down the examiner's contact information saying the patient would return our call another patient (RC7) stated the (yoga) exercises aggravated his shoulder and caused pain (he had three cracked vertebrae in the lower back); another (RC9) stated the (std. ex.) exercises were going "fine" but did not feel there was an improvement in her condition, another (RC10) stated he had "fallen off a little bit" and it was "more work than he was getting out of it" (yoga).

For the patients whose schedule was pushed back by 2 weeks, one patient (RC1) stated that she discontinued with the (std. ex.) exercises because of her upcoming surgery, another (RC4) was recommended to stop the (yoga) exercises by his clinician because of pain, another (RC6) did not answer the call and finally the last patient (RC8, yoga group) stated that she was not a part of the study because of vacation. During the final or $6^{\text {th }}$ week follow up call post assessment testing was scheduled through email for one patient (RC2) since follow up phone calls were ineffective and another patient (RC6) contacted HULC to schedule post assessment testing. The three patients who did not undergo pre/post testing (RC7/9/10) were asked specific questions regarding their perceptions of the video.

\section{Post intervention interview}

A semi structured interview developed by DM and approved by the 
second co author for the purpose of this study was used to interview patients (RC2 and RC6) one-on-one regarding their experiences of using the online exercise program. Interviews took place at HULC and patient answers were jotted by hand as well as audio recorded (using an application called 'Audio Recorder') on a cellular phone. Diary forms were collected and kept on file. The three patients who did not undergo pre or post testing (RC7/9/10) were asked the same questions that were asked of RC2 and RC6-except for one would you say your condition improved? Again answers given were jotted down by hand. However patient answers were not recorded. Patients who opted out of the study were asked for their general views about the online exercise program either during follow-up calls or through email but no concrete set of questions was created.

\section{Results}

\section{Patient demographics}

There were five female and five male patients in the study the youngest being 49 years old and the oldest being 68. Among the females the average age was 61 years and 59 among the males. Patient's group allocation (yoga or std. ex.) priority (level of urgency for surgery), age, sex, affected shoulder (left or right), RC history (number of RCI occurrences), and any co-interventions are provided below (Table 1).

Table I Key information of patients in study

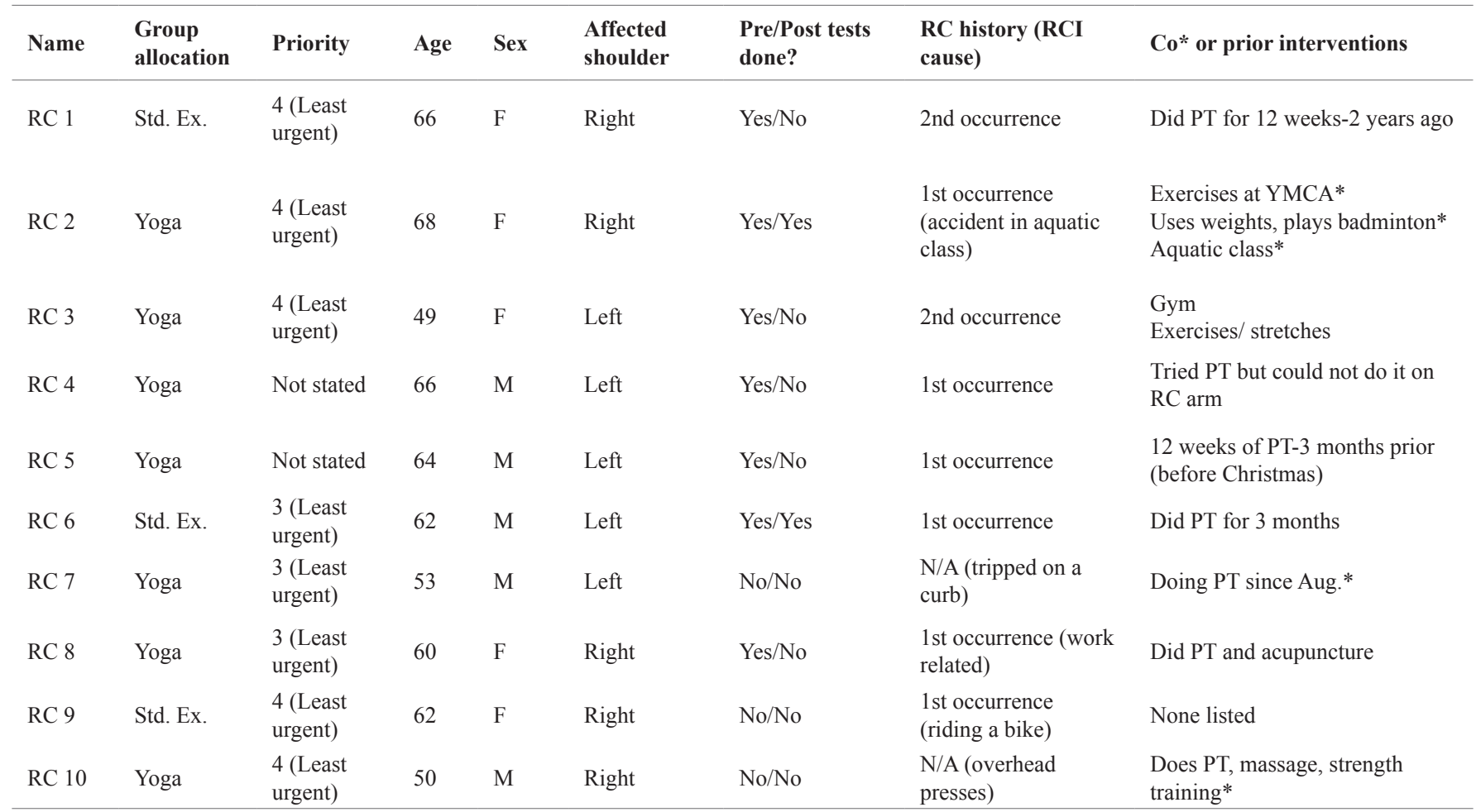

Note:"Priority": I,2=most urgent; 3,4=least urgent

\section{Feasibility issues}

\section{Recruitment}

Recruitment was a major concern in this study as from a total of 51 potentially eligible only 10 were recruited. Even if retention were high this would mean that a study requiring 200 participants would need to be drawn from a sample of about 1000 patients. A variety of barriers to recruitment were identified including incomplete information and the patient files issues with patient concerns about the travel associated with the study and the fact that surgery was imminent. Patients declined to participate for other reasons such as lack of availability (patients did not answer calls), general lack of interest, and/or busy with other commitments.

Potential solutions to these recruitment issues are possible. There are multiple sources within the hospital to verify patient contacts and a site specific study coordinator present when referrals were received could contact referral sources for contact information. The major barrier was the burden of interim assessments. Options for future studies include measuring ROM using video motion technology like Dart fish ${ }^{22}$ or photographs. ${ }^{23}$ Since the benefits of independent web/ video exercise programs are aimed at reducing patient burden in travel it appears that reducing visits for assessment for study outcomes is also important. Highlighting the potential convenience then may be important for recruitment since a number of potential participants were "not interested".

\section{Retention}

Retention was equally problematic with only 2 out of 10 completing the entire protocol (thus $80 \%$ drop out rate). While we asked non-completers to provide feedback only three provided qualitative feedback and five opted out at various points throughout the study primarily due to pain. This may need to factor in the issue that greater supervision and feedback may be needed to avoid 
increasing pain or poor targeting of the exercise program. In three cases we offered non attendees the option to perform the video-based exercises and provide qualitative feedback at the end and so better understand feasibility given our recruitment challenges. Regardless of this alternative option, certain patients still opted out. Potential ways to increase recruitment in future trials could be to offer an incentive and/or conduct in-house visits for assessment.

\section{Future sample size considerations}

Knowing that the clinically important difference (CID) or the smallest change that is meaningful for a patient ${ }^{6}$ for our group is smaller than the CID estimated for individuals we can assume that a potentially relevant CID for a future clinical trial might be a minimum four points on the SPADI ( $50 \%$ of the CID for individuals which ranges from 8 to 13$).{ }^{6}$ Calculating the sample size requirements for $80 \%$ power, $\alpha=0.05$ for patients with moderate disability (SPADI 59 vs. 50 suggests that 63 patients would be needed per group. Without improvements in recruitment and retention the sample size for one group would be achieved by screening 315 patients and enrolling 63 patients. Clearly the study is not feasible unless both improvements in recruitment and retention are attained.

\section{Outcome measures}

There is a possibility that some lack of standardization was introduced into the study protocol in terms of how strength and range of motion were measured since a consistent joint position was not used for all muscle testing. Better standardization of procedures might improve consistency across raters and overtime. Use of a single evaluator would reduce measurement errors but may not be possible if the sample size requirements necessitate a multicenter study. Standardized isometric testing that provides reliable results have been previously shown using a HHD for rotator cuff muscles. ${ }^{24}$ Lack of standardization weakens reliability and heightens random error, by making it difficult to determine a true change in patients over time. ${ }^{25}$

\section{Exercise fidelity and adherence}

The fidelity or ability of the patients to reproduce the intended exercises is unknown the program was designed to be delivered remotely and executed independently. While every intention to provide clear instructions was made, the extent to which these were understood and executed is unknown. Given the observations in this study and our previous case reports stating that exercises may have adverse consequences the need for greater supervision in future interventions is indicated. A potential solution to this would be to provide in person training at the beginning of the program and ensure that patients can reproduce the intended exercises prior to continuing on independently. A teach back approach to instruct patients on exercises and a check of the initial understanding could have avoided lack of exercise fidelity, may have enhanced satisfaction and in turn adherence ${ }^{26}$ but this would require more resources for an independent self management approach.

Our results suggest that for the two patients who completed the intervention adherence was very high. This is inconsistent with the number of people who dropped out of the study. Since this study used self reported measures (SPADI and Diary form) it is possible a bias is present. Patients were asked to be completely honest in their Diary and SPADI forms but it is possible patients may have felt the need to present a certain image thereby providing exaggerated/inaccurate information. Future studies should consider standardized adherence measures that are self-reported, can potentially wearable sensors that could objectively measure performance. In designing future trials it will be necessary to alter any shortcomings that were apparent in this study design.

In designing future trials, it will be necessary to alter any shortcomings that were apparent in this study design (Table 2).lysis

The feasibility of running a 6 week online exercise at home intervention in this study was weak given the high attrition rate and the counterproductive results for one patient. Adherence to the intervention was assessed through Diary forms which were closely observed to assess the days and times of when patients participated in the intervention. However only two such forms were analyzed and both showed excellent adherence.

Responses to the exercise programs were assessed through patient feedback which was given by email over-the-phone or in-person. Table 3 below demonstrates responses from the three patients who did not undergo pre/post testing. "Q.1, Q. 2" etc. correspond with the questions below.

1. How did you find the video exercises?

2. Did you do them every day for 5 minutes?

3. Would you say your condition improved?

4. What did you like about the study?

5. Is there anything you disliked about the study?

6. Is there any feedback you would like us to know?

Two of the patients who dropped out of the study informed the examiner via e-mail that the video was clear and the instructions were easy to follow. However, they discontinued because of pain.

Potential improvements in treatment implementation were assessed by patient feedback and analyzing the reasons why patients declined to be in the study assessed recruitment issues. The feasibility of implementing the outcome measures (ROM, strength and function) was attainable. Results were collected from a goniometer, HHD and SPADI and analyzed via percent change calculations. A simple mathematical formula was used: [(post result-pre result)/(pre result) $] * 100$. Moreover, SPADI forms specifically were analyzed by summing each subscale (pain and disability) as a percentage. The total SPADI score was calculated by summing questions from both subscales and dividing by the total.

Note: SPADI is out of 130, but if a question was left unanswered then the total became $120 .{ }^{4}$ Basic arithmetic (addition and subtraction) was done to determine if a change occurred from pre to post assessments. 
Table 2 Concerns in study and their rectification

\section{Error/Concern}

Video link not received by some patients

Patients may not be doing the exercises correctly.

Randomization. Too many patients were randomized to the yoga group

Yoga video does not stress the importance of engaging cuff muscles.

Patients could not attend pre/post testing due to location

Discordance between high reported adherence and low participation in the study

\section{Potential rectification}

Call to verify if patients received the video soon after it is first sent by the examiner.

Use of a hospital-based website and email to deliver interventions

Consider using a teach back approach at the initiation of the program that would be delivered in person, or a virtual one on one consultation with the therapist

Institute an adverse event reporting procedure to immediately contact patients who experience worsening of symptoms

Provide online tools for how patients can assess their own exercise fidelity

Block randomization. I.e. using block of six, so three patients get yoga and three patients get standard exercise treatment.

Stress the importance of cuff muscles in video by demonstrating the poses. (Perhaps show a right and wrong approach so patients have better under-standing).

Consider other alternatives that might be more important than yoga for future clinical trials such as different formats for providing standardized exercise

Basic science studies investigating whether yoga poses do engage rotator cuff muscles

Have a virtual pre/post testing environment or do in-house assessments

Consider alternate forms of measuring adherence including self-report and sensor-based

Table 3 Feedback from patients who did not undergo pre/post testing

\begin{tabular}{|c|c|c|c|c|c|c|}
\hline Patient & Q. 1 & Q. 2 & Q. 3 & Q. 4 & Q. 5 & Q. 6 \\
\hline $\mathrm{RC7}$ & $\begin{array}{l}\text { "Didn't find } \\
\text { them too bad" } \\
\text { "They would } \\
\text { definitely be a } \\
\text { benefit" }\end{array}$ & $\begin{array}{l}\text { Every day for } 6 \\
\text { weeks }\end{array}$ & $\begin{array}{l}\text { "I think so, yes" } \\
\text { "Definitely" }\end{array}$ & $\begin{array}{l}\text { "They were easy to } \\
\text { follow" } \\
\text { "Very well put } \\
\text { together and clear" }\end{array}$ & $\begin{array}{l}\text { First time using } \\
\text { it, nothing to } \\
\text { com-pare the } \\
\text { program to }\end{array}$ & Not at this point \\
\hline \multirow[b]{2}{*}{ RC9 } & "Helpful" & & & "Independent" & Validity concern & "Too loosely goosey" \\
\hline & $\begin{array}{l}\text { Videos were } \\
\text { clear }\end{array}$ & N/A & "Yes I would" & $\begin{array}{l}\text { "Didn't have to report } \\
\text { to any clinic/ person" }\end{array}$ & $\begin{array}{l}\text { No one checked } \\
\text { in } \\
\text { No pre/ post }\end{array}$ & $\begin{array}{l}\text { ROM and strength } \\
\text { was not measured }\end{array}$ \\
\hline $\mathrm{RC} 10$ & $\begin{array}{l}\text { "I found them } \\
\text { pretty basic" } \\
\text { (He did a lot } \\
\text { more) }\end{array}$ & $\begin{array}{l}\text { Did exercises } 2 \text { or } \\
3 \mathrm{x} \text { a week for the } \\
\text { first } 2 \text { weeks } \\
\text { Week } 3=\text { everyday } \\
\text { Weeks } 4-6=2 x / \text { day } \\
\text { At the start he had } \\
\text { a harder time due to } \\
\text { injured wrist }\end{array}$ & $\begin{array}{l}\text { "I don't think it } \\
\text { would hurt" }\end{array}$ & $\begin{array}{l}\text { "Pretty simple basic } \\
\text { pro-gram" }\end{array}$ & Not really & $\begin{array}{l}\text { "Hard to tell if the } \\
\text { exercises did anything } \\
\text { for the shoulder" }\end{array}$ \\
\hline
\end{tabular}




\section{Acknowledgements}

A sincere thank you to Prof. Joy Macdermid who provided her expertise throughout the making of this research study and in writing it. Thank you to Prof. Jackie Sadi for her valuable feedback and Dr. Ken Faber for his guidance. To Joshua Vincent and Katrina Munro at the Hand and Upper Limb Centre for their help with patient recruitment and testing. To the patients who provided their time and feedback massive thank you. Finally to my family and the Lord for showing me the way this far thank you for everything.

\section{Conflict of interest}

The author declares no conflict of interest.

\section{References}

1. Killian SE, Sarah EPT, Cavanaugh JT, et al. Rehabilitation guidelines after rotator cuff repair. Techniques in Shoulder \& Elbow Surgery. 2014;15(1):8-12.

2. Colvin AC, Egorova N, Harrison AK, et al. National trends in rotator cuff repair. J Bone Joint Surg Am. 2012;94(3):227-233.

3. Fleming JA, Seitz AL, Ebaugh DD. Exercise protocol for the treatment of rotator cuff impingement syndrome. $J$ Athl Train. 2010;45(5):483-485.

4. Breckenridge JD, McAuley JH. Shoulder pain and disability index (SPADI). J Physiother. 2011;57(3):197.

5. Ekeberg OM, Holter BE, Tveitå EK, et al. Agreement, reliability and validity in 3 shoulder questionnaires in patients with rotator cuff disease. BMC Musculoskelet Disord. 2008;9(1):68.

6. Roy J, Ma B, MacDermid JC, et al. Measuring shoulder function: A systematic review of four questionnaires. Arthritis Rheum. 2009;61(5):623-632.

7. Devet HC, Terwee CB, Ostelo RW, et al. Minimal changes in health status questionnaires: distinction between minimally detectable change and minimally important change. Health Qual Life Outcomes. 2006;4(1):54.

8. Celik D, Dirican A, Baltaci G. Intrarater reliability of assessing strength of the shoulder and scapular muscles. J Sport Rehabil. 2012;3:1-5.

9. Thorborg K, Petersen J, Magnusson SP, et al. Clinical assessment of hip strength using a hand-held dynamometer is reliable. Scand $\mathrm{J} \mathrm{Med} \mathrm{Sci}$ Sports. 2010;20(3):493-501.

10. Kolber M J, Cleland JA. Strength testing using hand-held dynamometry. Physical Therapy Reviews. 2005;10(2):99-112.

11. Andrews AW, Thomas MW, Bohannon RW. Normative values for isometric muscle force measurements obtained with hand-held dynamometers. Phys Ther. 1996;76(3):248-259.

12. Ciesla N, Dinglas V, Fan E, et al. Manual muscle testing: A method of measuring extremity muscle strength applied to critically ill patients. $J$ Vis Exp. 2011;12(50)
13. Beshay N, Lam PH, Murrell GAC. Assessing the reliability of shoulder strength measurement: Hand $\square$ held versus fixed dynamometry. Shoulder \& Elbow. 2011;3(4):244-251.

14. May LA, Burnham R S, Steadward RD. Assessment of isokinetic and hand-held dynamometer measures of shoulder rotator strength among individuals with spinal cord injury. Arch Phys Med Rehabil. 1997;78(3):251-255.

15. Wikholm J B, Bohannon RW. Hand-held dynamometer measurements: Tester strength makes a difference. J Orthop Sports Phys Ther. 1991;13(4):191-198.

16. Kelly BT, Kadrmas WR, Speer KP. The manual muscle examination for rotator cuff strength: An electromyographic investigation. Am J Sports Med. 1996;24(5):581-585.

17. Nussbaumer S, Leunig M, Glatthorn JF, et al. Validity and test-retest reliability of manual goniometers for measuring passive hip range of motion in femoroacetabular impingement patients. BMC Musculoskelet Disord. 2010;11:194.

18. Sabari JS, Maltzev I, Lubarsky D, et al. Goniometric assessment of shoulder range of motion: Comparison of testing in supine and sitting positions. Arch Phys Med Rehabil. 1998;79(6):647-651.

19. https://assessmentandinterventiongroup8.wordpress.com/rom/normal$\mathrm{rom} /$

20. Millett PJ, Warth RJ. Physical examination of the shoulder: An evidencebased approach. New York, USA: Springer; 2015.

21. Cools A, De Wilde L, Van Tongel A, et al. Measuring shoulder external and internal rotation strength and range of motion: Comprehensive intra-rater and inter-rater reliability study of several testing protocols. $J$ Shoulder Elbow Surg. 2014;23(10):1454-1461.

22. Khadilkar L, MacDermid J, Sinden K, et al. An analysis of functional shoulder movements during task performance using dartfish movement analysis software. s. 2014;8(1):1-9.

23. Crasto JA, Sayari AJ, Gray RR, et al. Comparative analysis of photograph-based clinical goniometry to standard techniques. Hand ( $N$ Y). $2015 ; 10(2): 248-253$.

24. Kolber MJ, Beekhuizen K, Cheng MS, et al. The reliability of hand-held dynamometry in measuring isometric strength of the shoulder internal and external rotator musculature using a stabilization device. Physiother Theory Pract. 2007;23(2):119-124.

25. http://www.nets.nihr.ac.uk/glossary?result_1655_result_page=F

26. Lis TW. Teach-back for quality education and patient safety. Urol Nurs. 2013;33(6):267-271,298. 